\title{
Three dimensional characterisation of electrical trees
}

DOI:

10.1109/CEIDP.2013.6748152

Link to publication record in Manchester Research Explorer

\section{Citation for published version (APA):}

Schurch, R., Rowland, S. M., Bradley, R. S., \& Withers, P. J. (2013). Three dimensional characterisation of electrical trees. In Annual Report - Conference on Electrical Insulation and Dielectric Phenomena, CEIDPIAnnu. Rep. Conf. Electr. Insul. Dielectr. Phenom. CEIDP (pp. 494-497) https://doi.org/10.1109/CEIDP.2013.6748152

\section{Published in:}

Annual Report - Conference on Electrical Insulation and Dielectric Phenomena, CEIDP|Annu. Rep. Conf. Electr. Insul. Dielectr. Phenom. CEIDP

\section{Citing this paper}

Please note that where the full-text provided on Manchester Research Explorer is the Author Accepted Manuscript or Proof version this may differ from the final Published version. If citing, it is advised that you check and use the publisher's definitive version.

\section{General rights}

Copyright and moral rights for the publications made accessible in the Research Explorer are retained by the authors and/or other copyright owners and it is a condition of accessing publications that users recognise and abide by the legal requirements associated with these rights.

\section{Takedown policy}

If you believe that this document breaches copyright please refer to the University of Manchester's Takedown Procedures [http://man.ac.uk/04Y6Bo] or contact uml.scholarlycommunications@manchester.ac.uk providing relevant details, so we can investigate your claim.

\section{OPEN ACCESS}


This is the accepted manuscript, which has been accepted by IEEE for publication (C) 2013 IEEE. Personal use of this material is permitted. Permission from IEEE must be obtained for all other uses, in any current or future media, including reprinting/republishing this material for advertising or promotional purposes, creating new collective works, for resale or redistribution to servers or lists, or reuse of any copyrighted component of this work in other works. The full reference is:

\section{'Three dimensional characterisation of electrical trees'}

R Schurch, S. M. Rowland, R S Bradley and P J Withers

IEEE Conference on Electrical Insulation and Dielectric Phenomena, Shenzhen, pp. 494-497 (2013)

DOI: $\underline{\text { 10.1109/CEIDP.2013.6748152 }}$ 


\section{Three dimensional characterisation of electrical trees}

\author{
Roger Schurch ${ }^{1}$ and Simon M. Rowland \\ School of Electrical and Electronic Engineering \\ The University of Manchester \\ Manchester, United Kingdom \\ s.rowland@manchester.ac.uk
}

\author{
Robert S. Bradley and Philip J. Withers \\ School of Materials \\ The University of Manchester \\ Manchester, United Kingdom
}

\begin{abstract}
Most of the geometric analysis of the structure of electrical trees has been performed using a two-dimensional (2-D) approach. However, electrical trees grow three-dimensionally (3D). The authors have recently shown that electrical trees can be 3-D imaged. A 3-D virtual replica can be created and through it, the tree characteristics analysed. Laboratory created electrical trees in polymeric insulation were scanned using $X$-ray computed tomography (XCT) for 3-D model reconstructions. Here global parameters such as diameter of tree channels, tree surface and volume are determined. The fractal dimension from 2-D projected patterns are shown to be lower than the fractal dimension from the entire 3-D model for both bush and branchtype trees. Local parameters such as numbers of channels, area covered by them and proportion of area degraded at a given cross-section are also calculated. Using the skeleton for analysing the tree structure, indices such as number of vertices, segment length, vertex density, tortuosity and branch angle are presented. Among them, vertex density provides a marked difference in value between a bush and a branch-type electrical tree. Using this 3-D approach for analysis achieves a more complete insight into treeing phenomena.
\end{abstract}

\section{INTRODUCTION}

A main mechanism of failure of polymeric high voltage insulation is electrical treeing. Electrical trees are well known artefacts of ageing in polymers, readily grown in the laboratory under high electrical stresses. Imaging these artificially created electrical trees has been an important tool for studying the phenomenon and getting a deeper understanding of how trees develop [1]. The most common imaging techniques are optical microscopy, scanning electron microscopy (SEM) and transmission electron microscopy (TEM). These techniques have the limitation of providing only a two-dimensional (2-D) view of the feature, therefore, loosing information that may be valuable in the analysis. We have shown that electrical trees can be three-dimensionally (3-D) imaged. Virtual replicas of electrical trees can be created using X-ray computed tomography (XCT) [2] or Serial Block-Face SEM (SBFSEM) [3]. Through them, the entire 3-D structure can be analysed and new quantification tools are now available. In this paper, we exploit the 3-D data obtained by XCT for structural quantification of electrical trees.

\section{Tree structure characterisation and quantification}

Conventionally, using optical means, electrical trees have been characterised by their 2-D shape [4-6], length [4, 6, 7], growth rate $[5,8]$, ratio of the width and the length [4] and fractal dimension [4, 6-9]. Fractal dimension has become in some degree a standard quantifier for characterisation of the structure (shape) of the electrical tree. It has been stated in [10] that the fractal dimension $\left(D_{f}\right)$ for branch-type patterns is within the range of $1<\mathrm{D}_{\mathrm{f}}<2$ and for bush-type structures $2<$ $\mathrm{D}_{\mathrm{f}}<3$. This parameter is important since it is related to the tree growth rate: a lower fractal dimension (branch-type) results in shorter time to breakdown $[6,8]$.

Fractal dimension has typically been calculated using 2-D projected patterns due to the convenience of obtaining 2-D images of the tree. Only a few attempts of estimating fractal dimension from 3-D patterns of real electrical trees (not simulated) have been carried out; a review of them is found in [9]. Those studies used optical serial sectioning and optical computed tomography for 3-D reconstruction of the tree. The authors pointed out that for dense (bush) electrical trees, the fractal dimension of 2-D projected patterns differs from that of the complete 3-D pattern, because the overlapping in the 2-D projection. Also, worth noting is that not only the amount of 3$\mathrm{D}$ data reported is scarce, but also that the resolution provided by the imaging techniques used at that time $(10 \mu \mathrm{m} / \mathrm{px}$ at the best using sectioning method) is considerably less that the resolution acquired by the techniques available now, such as XCT presented here (around one micrometre).

Using a 3-D approach with the XCT and SBFSEM imaging techniques, we have characterised electrical trees through global parameters (of the entire tree) such as mean diameter of the tree channels, tree volume and proportion of volume degraded [2, 3]; and local parameters (considering a crosssection) such as number and area of tree channels, and proportion of area degraded.

Tree structures are encountered in the human body too. For example, bronchial and vascular networks are tree-like structures that have been characterised for modelling and diagnosis purposes [11, 12]. The tree geometry has been quantified by indices such as number of segments or branches, length of the branches, branch angle, ratio of branch length to diameter, ratio of diameter to parent diameter and ratio of length to parent length, amongst others. However, vascular networks are different to electrical trees as their function is to transport fluids, and therefore, the structure is governed by the idea of feeder and receiver, where a parent vessel is larger in diameter than a child vessel, questioning the usefulness of some of these parameters in their application to electrical trees. Nevertheless, some of these 'biomedical indices' are calculated in this paper for further evaluation of electrical trees.

\footnotetext{
${ }^{1}$ R. S. is also with Universidad Tecnica Federico Santa Maria, Valparaiso, Chile
} 


\section{METHODOLOGY}

Samples were prepared using the conventional point-toplane configuration with a gap of $\sim 2 \mathrm{~mm}$ between the needle tip and the bottom of the sample. Electrical trees were grown until the desired tree length was reached. Afterwards, the samples were machined to create a $2-3 \mathrm{~mm}$ diameter cylinder of polymer containing the tree to be imaged by the XCT scan. For an explanation of tomography procedures refer to [2].

\section{A. Sample 1}

A bush-type electrical tree was created in a transparent polymer and XCT-imaged as described in [2]. The reconstruction of the tree resulted in a total of 512 cross-section slices with a voxel (volumetric pixel) size of $1.07 \mu \mathrm{m}$ and the feature was image-segmented using Avizo 7.1 software (VSG, Burlington, MA, USA). For the analysis and quantification, a selection of 356 slices (381 $\mu \mathrm{m})$ was considered.

\section{B. Sample 2}

The sample consisted of an epoxy resin cube (Huntsman Araldite ${ }^{\circledR}$ LY5052 - Aradur ${ }^{\circledR}$ HY5052) of 25 mm edge with an acupuncture needle inserted (Hwato ${ }^{\circledR} 0.35 \mathrm{~mm}, \sim 5 \mu \mathrm{m}$ tip radius). The sample was stressed under $10 \mathrm{kVrms} 50 \mathrm{~Hz}$ until the electrical tree was optically noticeable. The resulting feature was a branch-type electrical tree of around $200 \mu \mathrm{m}$ length. The sample was scanned for $60 \mathrm{~h}$ using an Xradia nanoXCT-100 microscope at $150 \mathrm{~nm}$ resolution, during which time 721 radiographs were taken over $180^{\circ}$. Due to the limited field of view of the system $(\sim 60 \mu \mathrm{m})$, the tree was not entirely imaged. The feature was reconstructed with a voxel size of 0.13 $\mu \mathrm{m}$ and segmented using Avizo software for 3-D rendering and further analysis. A total of 325 slices, giving $42.1 \mu \mathrm{m}$ length along the tree were used for the analysis.

\section{Sample 3}

A similar procedure as for Sample 2 was followed for Sample 3, using the same material and needle type. The same stress voltage was applied and removed as soon as an electrical tree was perceived, aiming to create a small tree. The resulted feature was an electrical tree less than $100 \mu \mathrm{m}$ of length that was scanned using an Xradia VersaXRM-510. For the tomography scan, which took 22 h, 1501 radiographs were taken over $360^{\circ}$. The voxel size of the reconstruction was 0.37 $\mu \mathrm{m}$ and 160 slices (giving a $59 \mu \mathrm{m}$ section) were selected for the 3-D model creation and calculations, described in the following section.

\section{Calculation of indices for electrical tree characterisation}

With the 3-D model created, electrical tree characteristics were quantified. Considering the entire electrical tree, global parameters were calculated, including the mean diameter of the tree channels using the local thickness plugin for ImageJ software. Surface area, volume and convex hull volume of the tree were measured using Avizo. The convex hull is the smallest convex polyhedral surface that contains the entire feature [3]. From these, two indirect parameters were calculated: the surface-to-volume ratio, and the ratio between the volume and the convex hull volume, which was termed 'proportion of volume degraded'. Furthermore, the fractal dimension of the tree structure was estimated using the box counting method in Matlab. According to the method, the value reported is the slope of the logarithmic plot of the number of boxes against the reciprocal of the size of the box that was used to fill the feature. This was also calculated in 2-D for comparison by projecting the 3-D virtual image onto the three orthogonal planes $(x y, x z, y z)$, where $z$ is the axial direction of the tree, parallel to the needle. Local parameters were developed by considering $x y$ cross-sections through the tree to investigate the change in the tree structure along its axial direction. The number, area and 2-D convex hull area of tree channels in each slice were determined using Matlab. In addition, the proportion of area degraded was defined as the ratio between the channels area and the convex hull area.

Interconnected structures can also be analysed as networks by generating a 'skeleton', consisting of line segments following the centres of the channels connected at branch points (vertices). The number of vertices and segments, as well as the mean segment length between branch points were calculated. Vertex density was defined as the ratio between the number of vertices and the convex hull volume. Additional characteristics were calculated including the 'end point fraction', which is the proportion of vertices which denote end points of the channels (with the scan volume) rather than branch points; branching order, which is the average number of segments connected to each vertex (the branch order must be $\geq 3$ in order for the vertex to be a branch point); and branch angle, which is the mean angle between line segments at a branch point. The branch angle was derived by first specifying a 'parent' line segment for a given vertex, this being the line segment that is reached first when traversing the tree from the start point of the tree. The vector angle between the beginning sections of the parent and each child line-segment was then calculated. The 'straightness' of paths through each tree was measured by calculating the tortuosity of the shortest paths from the tree starting point to each end point, with the tortuosity being the ratio between the length along the route
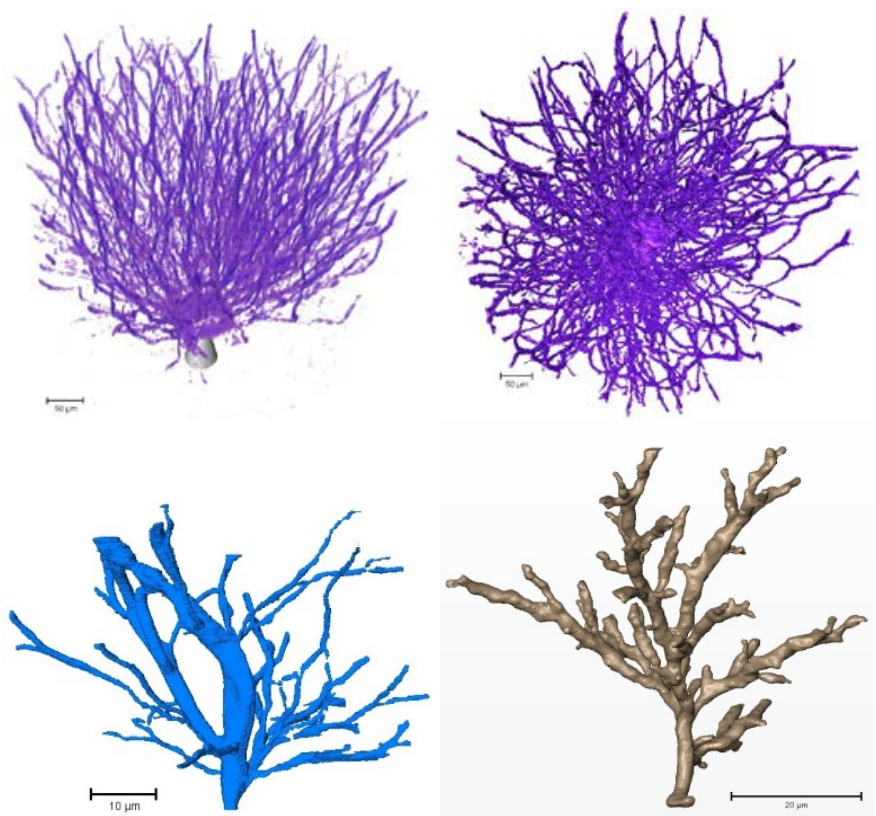

Fig. 1. Virtual replica of the samples using XCT. Top: two viewing angles of Sample 1 (Scalebar: $50 \mu \mathrm{m}$ ) [2]. Bottom-left: Sample 2 (Scalebar: $10 \mu \mathrm{m}$ ). Bottom-right: Sample 3 (Scalebar: $20 \mu \mathrm{m}$ ) 
and the Euclidean distance from the start to end point. Finally, the ratio between the length and the diameter of the branches was calculated.

\section{RESULTS AND DISCUSSION}

Three-dimensional renderings of the electrical trees are shown in Fig. 1. Animations of the cross-sections (slices) along the progression of the tree and virtual animations with the 3-D replica axially rotating can be seen in [13].

The results of the calculations for electrical tree characterisation are summarised in Table 1. Values calculated over a set of points giving a frequency distribution are presented in the form of 'mean value \pm standard deviation'. While it should be noted that different polymers, stress times and scan settings were used, it is reasonable to compare and evaluate the parameters for characterisation and structural discrimination, as Sample 1 represents a relatively large bushtype tree, while Samples 2 and 3 are relatively small branchtype trees. The mean diameter of the tree channels were similar for Sample 2 and $3(\sim 2 \mu \mathrm{m})$, but larger for Sample $1(\sim 4 \mu \mathrm{m})$. However, this difference may be attributable to the polymer and stress time. In addition, the imaging resolution (related to the voxel size) makes the diameter value for Sample 1 less precise. It can also be noticed that the vertex density was around one order of magnitude different between the two groups of samples (Sample 1 vs. Sample 2 and 3). Other indices useful for classification may be the end point fraction ( 0.2 vs. 0.5 ) and the degraded volume (e.g. accumulated up to $30 \mu \mathrm{m}$ gave almost two order of magnitude difference). The average value of $\sim 3$ for branching order reveals that normally a branch splits into two new branches (' $\mathrm{Y}$ ' structure); the branch angle of that bifurcation is around $65^{\circ}$ (relative to the parent branch, or $\sim 130^{\circ}$ between child branches). It is interesting to observe that the segment-length-to-diameter ratio was virtually constant amongst the samples.

Fractal dimension values from the 3 -D patterns were in accordance with the conventional classification for branch or bush tree (fractal dimension below 2 for branch-type tree). However, for 2-D projected patterns that criterion did not apply in the cases analysed here. In [9] it was suggested that for a high fractal dimension tree (bush-type) the 2-D value is lower than the 3-D, because the loss of information in the projection; but, for a low fractal dimension tree (branch-type), the 2-D value should be similar to the $3-\mathrm{D}$ one. However, the results obtained here showed that the fractal dimensions from the 2-D projected patterns are lower than the real 3-D values in all cases (11-16\%). This confirms that it is essential for the electrical tree to be analysed in 3-D for realistic values to be obtained.

Additionally, local parameters were measured on a slice that was $30 \mu \mathrm{m}$ distant from the tree starting point (where data was gathered for Table 1). As expected, a noticeable difference in channels' areas and accumulated degraded volume was observed between Sample 1 and Samples 2 and 3.

A cross-sectional analysis was carried out as is depicted in Fig. 2, where the number of electrical tree channels (continuous line) and the proportion of area degraded (dashed line) for each sample is plotted as a function of the distance from the tree starting point. For Sample 2, the values are shown from $5 \mu \mathrm{m}$ to obtain better resolution in the plot. The proportion of area
Table 1. Quantification of electrical trees

\begin{tabular}{|c|c|c|c|}
\hline & Sample 1 & Sample 2 & Sample 3 \\
\hline Slices analysed (length $[\mu \mathrm{m}]$ & $356(381)$ & $325(42)$ & $160(59)$ \\
\hline Voxel size $[\mu \mathrm{m}]$ & 1.07 & 0.13 & 0.37 \\
\hline \multicolumn{4}{|c|}{ Global parameters } \\
\hline Diameter tree channels $[\mu \mathrm{m}]$ & $4.4 \pm 1.7$ & $1.9 \pm 1.0$ & $2.0 \pm 0.4$ \\
\hline Tree volume $\left[\mu^{3}\right]$ & $1.78 \times 10^{6}$ & $1.30 \times 10^{3}$ & $1.62 \times 10^{3}$ \\
\hline Convex hull volume $\left[\mathrm{m}^{3}\right]$ & $5.06 \times 10^{7}$ & $6.98 \times 10^{4}$ & $5.62 \times 10^{4}$ \\
\hline Prop. volume degraded & $3.5 \%$ & $1.9 \%$ & $2.9 \%$ \\
\hline Surface area $\left[\mu^{2}\right]$ & $1.70 \times 10^{6}$ & $3.83 \times 10^{3}$ & $3.50 \times 10^{3}$ \\
\hline Surface/volume ratio $\left[\mu^{-1}\right]$ & 0.95 & 2.95 & 2.16 \\
\hline \multicolumn{4}{|c|}{ Fractal dimension } \\
\hline $3-\mathrm{D}$ & 2.2 & 1.9 & 1.7 \\
\hline 2-D xy projected & 1.8 & 1.7 & 1.4 \\
\hline 2-D xz projected & 1.9 & 1.7 & 1.5 \\
\hline 2-D yz projected & 1.8 & 1.7 & 1.4 \\
\hline \multicolumn{4}{|c|}{ Skeletonisation } \\
\hline Number of vertices & 5040 & 174 & 78 \\
\hline Number of segments & 6191 & 164 & 78 \\
\hline End point fraction & 0.2 & 0.5 & 0.5 \\
\hline Branching order & $3.2 \pm 0.5$ & $3.0 \pm 0.2$ & $3.2 \pm 0.4$ \\
\hline Segment length $[\mu \mathrm{m}]$ & $17.3 \pm 16.1$ & $6.5 \pm 6.0$ & $7.3 \pm 4.4$ \\
\hline Vertex density $\left[\mu^{-3}\right]$ & $1.0 \times 10^{-4}$ & $2.5 \times 10^{-3}$ & $1.4 \times 10^{-3}$ \\
\hline Seg. length/diameter ratio & 3.93 & 3.42 & 3.67 \\
\hline Tortuosity & $1.6 \pm 0.6$ & $1.3 \pm 0.1$ & $1.2 \pm 0.1$ \\
\hline Branch angle [deg] & $64 \pm 30$ & $66 \pm 33$ & $69 \pm 27$ \\
\hline \multicolumn{4}{|c|}{ Local parameters at $30 \mu \mathrm{m}$} \\
\hline Number of channels & 17 & 13 & 6 \\
\hline Prop. area degraded & $6.7 \%$ & $2.3 \%$ & $7.8 \%$ \\
\hline Channels area $\left[\mu \mathrm{m}^{2}\right]$ & 700 & 28 & 34 \\
\hline Degraded volume $\left[\mu^{3}\right]$ & 79221 & 918 & 1059 \\
\hline
\end{tabular}

degraded approaches $100 \%$ close to the tree starting points of Sample 2 and 3, as well as towards the end of Sample 3, which is due to there being only a few tree channels, yielding to a convex hull area similar to the channel area. The scattering of the data in the plot of Sample 2 and 3 is mostly attributed to fewer features to count.

Further analysis was conducted by dividing Sample 1 into three sections to investigate the variation in the parameters. The entire stack of slices was divided in ratios of $15 \%$ (54 slices $58 \mu \mathrm{m}), 70 \%$ (249 slices - $266 \mu \mathrm{m}$ ) and 15\% (53 slices -57 $\mu \mathrm{m})$ for Section 1 (close to the tree starting point), Section 2 (middle part) and Section 3 (furthest from the starting point within the analysed volume), respectively. It has to be noted that Section 3 is not where the tree tips were located, is the last part of the data analysed in this paper. This is the reason why some index values were similar between Section 2 and 3. The original last part was cropped because the data were noisy, making it difficult to analyse. The results for the global parameters are summarised in Table 2. The mean diameter of the tree channels in Section 1 is almost double that of the other sections $(8.2 \mu \mathrm{m}$ vs. $4.2 \mu \mathrm{m})$ and moreover, the proportion of volume degraded is considerable greater $(13.3 \%$ vs. $3.8 \%$ and $2.0 \%$, for sections 1,2 and 3 , respectively). 

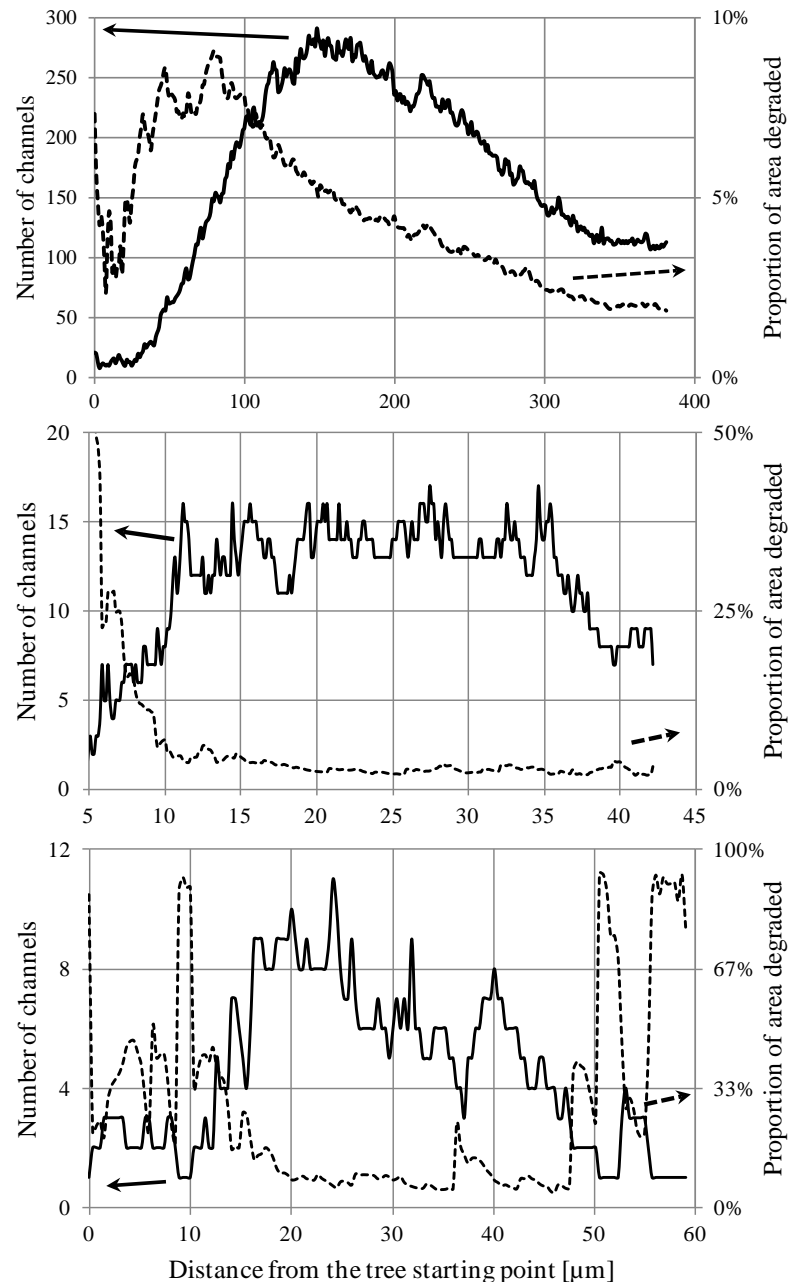

Fig. 2. Cross-section analysis of Sample 1 (top), Sample 2 (middle) and Sample 3 (bottom).

Table 2. Parameters for Sample 1 divided in sections.

\begin{tabular}{lccc}
\hline \multicolumn{1}{c}{ Sample 1: } & Sec. 1 & Sec. 2 & Sec. 3 \\
\hline \hline Diameter tree channels $[\mu \mathrm{m}]$ & $8.2 \pm 3.9$ & $4.2 \pm 0.8$ & $4.2 \pm 0.8$ \\
\hline Tree volume $\left[\mathrm{\mu m}^{3}\right]$ & $1.38 \times 10^{5}$ & $1.46 \times 10^{6}$ & $1.79 \times 10^{5}$ \\
\hline Convex hull volume $\left[\mathrm{\mu m}^{3}\right]$ & $1.04 \times 10^{6}$ & $3.82 \times 10^{7}$ & $9.14 \times 10^{6}$ \\
\hline Prop. volume degraded & $13.3 \%$ & $3.8 \%$ & $2.0 \%$ \\
\hline Surface area $\left[\mathrm{\mu m}^{2}\right]$ & $7.58 \times 10^{4}$ & $1.46 \times 10^{6}$ & $1.74 \times 10^{5}$ \\
\hline Surface/volume ratio $\left[\mathrm{\mu m}^{-1}\right]$ & 0.55 & 1.00 & 0.97 \\
\hline
\end{tabular}

\section{CONCLUSIONS}

Detailed characterisation of electrical trees with a 3-D perspective has been presented. Virtual replicas of electrical trees have been created using X-ray computed tomography from which conventional and new parameters were calculated and evaluated for structure discrimination. One bush-type and two small branch-type electrical trees were analysed. Global parameters such as diameter of tree channels, tree surface area and volume were found to be informative. The fractal dimension of the trees, as calculated in 3-D, was confirmed as being an important discriminator. However, the 2-D measurements of fractal dimension (derived from the projected patterns generated by the 3-D replicas) by comparison were in all cases (for both bush and branch type tree structures) considerably lower and potentially misleading. This stresses the importance of analysing electrical trees using a 3-D approach.

The electrical tree structure has also been analysed as a network through its skeleton. Indices were computed, such as number of vertices, segment length, vertex density, tortuosity and branch angle, amongst others. It was observed, as expected, that vertex density provided a marked difference between a

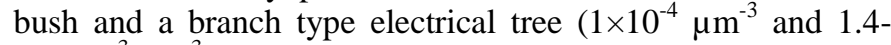

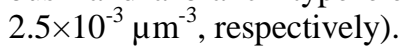

A more complete analysis is achieved when the 3-D approach is used, because it discloses the entire structure of the tree. The new parameters presented here for electrical tree characterisation add valuable information about the structure of the tree.

\section{ACKNOWLEDGMENT}

Roger Schurch would like to thank the Dielectrics and Electrical Insulation Society (IEEE-DEIS) for the Graduate Fellowship Award and the scholarship support of CONICYT (Chilean Research Council). Philip Withers would like to acknowledge funding from EPSRC for the Manchester X-ray Imaging Facility under EP/F007906/1 and EP/F028431/1.

\section{REFERENCES}

[1] R. Schurch, S. M. Rowland, and P. J. Withers, "Techniques for electrical tree imaging," in Imaging Systems and Techniques (IST), 2012 IEEE International Conference on, 2012, pp. 409-414.

[2] R. Schurch, S. M. Rowland, R. S. Bradley, and P. J. Withers, "A novel approach for imaging of electrical trees," in Electrical Insulation and Dielectric Phenomena (CEIDP), 2012 Annual Report Conference on, 2012, pp. 593-596

[3] R. Schurch, S. M. Rowland, and T. Starborg, "Serial Block-Face Scanning Electron Microscopy for Three- Dimensional Imaging of Electrical Trees," in International Conference on Solid Dielectrics (ICSD), 2013.

[4] Z. Xiaoquan and G. Chen, "Propagation mechanism of electrical tree in XLPE cable insulation by investigating a double electrical tree structure," Dielectrics and Electrical Insulation, IEEE Transactions on, vol. 15, pp. 800-807, 2008.

[5] M. Bao, X. Yin, and J. He, "Structure characteristics of electrical treeing in XLPE insulation under high frequencies," Physica B: Condensed Matter, vol. 406, pp. 2885-2890, 2011.

[6] C. Xiangrong, X. Yang, C. Xiaolong, S. J. Dodd, and L. A. Dissado, "Effect of tree channel conductivity on electrical tree shape and breakdown in XLPE cable insulation samples," Dielectrics and Electrical Insulation, IEEE Transactions on, vol. 18, pp. 847-860, 2011.

[7] A. Xie, X. Zheng, S. Li, and G. Chen, "Investigations of electrical trees in the inner layer of XLPE cable insulation using computer-aided image recording monitoring," Dielectrics and Electrical Insulation, IEEE Transactions on, vol. 17, pp. 685-693, 2010.

[8] G. Chen and C. Tham, "Electrical treeing characteristics in XLPE power cable insulation in frequency range between 20 and $500 \mathrm{~Hz}$," Dielectrics and Electrical Insulation, IEEE Transactions on, vol. 16, pp. 179-188, 2009.

[9] K. Kudo, "Fractal analysis of electrical trees," Dielectrics and Electrical Insulation, IEEE Transactions on, vol. 5, pp. 713-727, 1998.

[10] L. A. Dissado and J. C. Fothergill, Electrical Degradation and Breakdown in Polymers. London: Peter Peregrinus Ltd., 1992.

[11] M. H. Tawhai, P. Hunter, J. Tschirren, J. Reinhardt, G. McLennan, and E. A. Hoffman, "CT-based geometry analysis and finite element models of the human and ovine bronchial tree," Journal of Applied Physiology, vol. 97, pp. 2310-2321, 2004.

[12] Y. C. Lee, A. R. Clark, M. K. Fuld, S. Haynes, A. Divekar, E. A Hoffman, et al., "MDCT-based quantification of porcine pulmonary arterial morphometry and self-similarity of arterial branching geometry," Journal of Applied Physiology, February 28, 20132013.

[13] R. Schurch, S. M. Rowland, R. S. Bradley, and J. P. Withers. (2013, 11th June). Electrical Trees CEIDP. Available: http://www.youtube.com/user/ElectricalTreesCEIDP/videos 